\title{
Anterior pituitary trophic responses to dexamethasone withdrawal and repeated dexamethasone exposures
}

\author{
L A Nolan and A Levy \\ University Research Centre for Neuroendocrinology, Bristol Royal Infirmary, Lower Maudlin Street, Bristol BS2 8HW, UK \\ (Requests for offprints should be addressed to A Levy; Email: a.levy@bris.ac.uk)
}

\begin{abstract}
Glucocorticoid withdrawal, depending on the dose and duration of treatment, results in a transient but sometimes prolonged reduction in hypothalamo-pituitary-adrenal (HPA) axis secretory responsiveness. As the anatomic basis of HPA axis suppression remains uncertain, we have directly examined changes in trophic activity within the rat anterior pituitary gland following dexamethasone withdrawal and re-treatment. Treatment of adrenalectomised, male Wistar rats with dexamethasone results in a discrete, highly significant burst of apoptosis in the anterior pituitary with concurrent suppression of mitosis. Despite a surge in mitotic activity immediately after dexamethasone withdrawal, calculated total anterior pituitary cell populations remain below that seen in untreated adrenalectomised controls. Repeated exposures to dexamethasone show that the dexamethasone-sensitive cell
\end{abstract}

population that is deleted by apoptosis is partially but not completely restored. As the amplitude of apoptotic bursts induced by second and third dexamethasone exposures are similar but smaller than that induced by initial exposure, it appears that the very first exposure to dexamethasone deletes a subset of anterior pituitary cells that are either not restored at all, or are only replaced very slowly. The reduced proportion of corticotrophs contributing to the increase in mitotic index after dexamethasone withdrawal corroborates this. Although continued cell turnover within the pituitary predicts that the absolute cellular deficit would diminish with time, the effects seen may contribute to the delayed recovery of pituitary axis function following cessation of glucocorticoid treatment.

Journal of Endocrinology (2001) 169, 263-270

\section{Introduction}

The adaptive responses made by the anterior pituitary gland to altered physiological and/or pharmacological circumstances may encompass changes in the synthetic or secretory capacity of pre-existing cells, increased or decreased rates of cell division and cell death, changes in cell survival time, or transdifferentiation between mature cell phenotypes. Failure to return to the normal steady state equilibrium once a particular stimulus has passed may modify the response of the pituitary to subsequent stimuli or predispose the gland to further trophic anomalies.

Withdrawal of long-term corticosteroid treatment in both man (Graber et al. 1965, Harrison et al. 1982, Schlaghecke et al. 1992, Ford et al. 1997) and adrenalintact rats (Nicholson et al. 1984, Calogero et al. 1990, Huang 1994) has been shown to reduce temporarily the ability of each component of the hypothalamo-pituitaryadrenal (HPA) axis to respond to endogenous and exogenous stimuli. The rate of recovery of the individual components of the HPA axis vary and a number of factors influence restoration of appropriate responsiveness. These include the specific glucocorticoid used, the route and timing of its administration, the complement and integrity of glucocorticoid receptors of the individual concerned and the duration and dose of treatment. In man, the biochemical but not necessarily clinical recovery of the HPA axis following short courses of glucocorticoid treatment appears to be complete from between 5 and 10 days after each treatment (Carella et al. 1993). Chronic alternate-day glucocorticoid therapy results in relatively mild suppression of the HPA axis (Schurmeyer et al. 1985) but prolonged exposure at high levels, either through administration of pharmacological doses for immunosuppressive or anti-inflammatory effects, or endogenous supraphysiological levels in Cushing's disease, may cause HPA axis suppression lasting a year or more (Graber et al. 1965, Livanou et al. 1967, Harrison et al. 1982, Avgerinos et al. 1987, Schlaghecke et al. 1992, Ford et al. 1997). In intact male rats treated with dexamethasone for between 2 and 14 days, it has been shown that recovery of pituitary corticotrophs in terms of adrenocorticotrophin (ACTH) secretion occurs between 4 and 7 days after withdrawal of the glucocorticoid (Nicholson et al. 1984, Calogero et al. 1990, Huang 1994). Following 2 days of dexamethasone treatment, basal corticosterone levels return to normal 
within 2 days (Nicholson et al. 1984), but take 10 days to recover following a 14-day course of treatment (Huang 1994). In these studies there were no opportunities to examine the underlying trophic effects of glucocorticoid withdrawal and repeated dexamethasone treatment, although it is these effects, intuitively, that might be expected to give rise to the more long term secretory consequences of glucocorticoid treatment.

We have previously shown that two weeks after adrenalectomy, a dexamethasone treatment results in a discrete, highly significant burst of apoptotic activity in the rat anterior pituitary, together with suppression of mitotic activity that persists for the duration of treatment (Nolan et al. 1998). The effects of these changes in apoptotic and mitotic rates predict an absolute decline in anterior pituitary cell numbers such that total pituitary cell numbers fall to well below basal levels by the end of the two-week dexamethasone exposure. Although it is not possible to demonstrate definitively which subsets of pituitary cells are involved, this apoptotic activity provides one explanation for a potential reduction in corticotroph cell numbers after dexamethasone treatment, and a mechanism by which the corticosterone response to stress is potentially impaired, at least in the short term. The aims of the present study were, first, to investigate the role of trophic activity in the remodelling of the anterior pituitary after withdrawal of dexamethasone in both adrenalectomised and adrenalintact rats, and secondly, as dexamethasone causes apoptotic deletion of a population of cells, to determine to what extent a trophically active dexamethasone-sensitive cell population is restored after cessation of dexamethasone treatment.

\section{Materials and Methods}

\section{Animals and treatments}

All animal procedures were carried out in accordance with UK Home Office animal welfare regulations. Male Wistar rats weighing between 100 and $125 \mathrm{~g}$ were purchased from Bantin and Kingman Universal Ltd (Hull, Humberside, UK) and allowed to acclimatise for one week before being surgically adrenalectomised under anaesthesia with an intraperitoneal injection $(1 \mathrm{ml} / 100 \mathrm{~g}$ body weight) of a mixture of 2:2:2 tribromoethanol $(2 \% \mathrm{w} / \mathrm{v})$ in $100 \%$ ethanol $(8 \% \mathrm{v} / \mathrm{v})$ and 2-methylbutan-2-ol $(1 \cdot 2 \%$ $\mathrm{v} / \mathrm{v})$ in $0.9 \%$ saline. Adrenalectomised rats were given $0.9 \%$ saline to drink after surgery. After 2 weeks, dexamethasone $(0.75 \mu \mathrm{g} / \mathrm{ml}$; Sigma Chemical Co., Poole, Dorset, UK) was added to the saline or drinking water for up to 2 weeks. In addition, a subcutaneous injection of $20 \mu \mathrm{g} / 100 \mathrm{~g}$ body weight dexamethasone sodium phosphate in saline (David Bull Labs., Warwick, Warks, UK) was given between 1300 and $1400 \mathrm{~h}$ for the first 3 days of glucocorticoid treatment. Control animals received an equal volume of saline.
To examine the effects of glucocorticoid withdrawal on pituitary trophic activity, groups of adrenalectomised and control animals ( \pm dexamethasone treatment) were killed by stunning and decapitation at intervals from $24 \mathrm{~h}$ to 14 days following either surgery, or the start or withdrawal of dexamethasone treatment. Animals were killed immediately after removal from their cages in a separate room adjoining that in which they had been housed.

The trophic effects of repeated 3- or 14-day exposures to dexamethasone were evaluated after 1- or 2-week recovery periods respectively and compared with suitably prepared age-matched controls. A shorter treatment duration and recovery period was chosen to evaluate the effects of greater than 2 dexamethasone exposures for several reasons. First, all of our previous experiments showed no further trophic changes beyond one week after adrenalectomy. Secondly, no further trophic changes were seen after 3 days of dexamethasone treatment or more than 1 week after dexamethasone withdrawal. Thirdly, we were concerned that the age-related reduction in trophic activity would have confounded the results of more prolonged studies (Nolan et al. 1999). Apart from a small number of very closely spaced time points around predicted trophic events (where $n$ was $\geq 3$ ), the number of animals at each time point was at least 6 (see Fig. 1 for time points).

Preparation of tissue sections Immediately after decapitation, pituitary glands were carefully removed and fixed in $4 \%$ formaldehyde in phosphate-buffered saline for $48 \mathrm{~h}$. The tissue was then washed in two changes of fresh phosphate-buffered saline before being embedded in $1 \%$ agar and processed for paraffin wax embedding. A series of $2 \mu \mathrm{m}$-thick axial sections were cut from each pituitary for immunocytochemistry and histological analysis.

Immunocytochemistry Axial $2 \mu$ m-thick pituitary sections were deparaffinised in xylene and rehydrated through a graded series of alcohols followed by incubation in $0.1 \mathrm{M}$ phosphate buffer. Endogenous peroxidases were deactivated in phosphate buffer containing $20 \%$ methanol, $0 \cdot 2 \%$ Triton $\mathrm{X}-100$ and $1.5 \%$ hydrogen peroxide for $30 \mathrm{~min}$. Sections were blocked in 1\% normal horse serum and incubated overnight in a monoclonal mouse antiserum raised against ACTH residues 1-24 (1:200; Novacastra Labs Ltd, Newcastle upon Tyne, UK) at $4{ }^{\circ} \mathrm{C}$. After washing in three changes of phosphate buffer, the sections were incubated at room temperature for $4 \mathrm{~h}$ in peroxidaselabelled anti-mouse immunoglobulin G (1:200; Vector Labs, Peterborough, Cambs, UK). Negative control sections were incubated in the absence of either primary or secondary antibody. Immunoreactive cells were visualised using the $3^{\prime}, 3^{\prime}$-diaminobenzidine (DAB)/glucose oxidase/ glucose method as described by Shu et al. (1988). Nuclei were lightly counterstained with Harris's haematoxylin (Sigma). 


\section{Image analysis}

The histological markers that were used to identify apoptotic cells were clusters of two or more apoptotic bodies consisting of extremely dense round or oval structures varying in size from approximately 0.7 to $4 \mu \mathrm{m}$ and surrounded by normal cells. Earlier stages of apoptosis cannot be visualised using haematoxylin and eosin (H\&E) staining and light microscopy. Apoptotic and mitotic cell counts were performed on $2 \mu \mathrm{m}$-thick H\&E-stained rat pituitary sections at $1000 \times$ magnification with the aid of a dedicated real-time computer system to tag and tally the co-ordinates and trophic assignment of manually identified structures within each tissue section (Nolan et al. 1998). The computerised Highly Optimised Microscope Environment (AxioHOME, Zeiss (Brugal et al. 1992)) that was used, projects a virtual image of the computer screen which appears fractionally above the actual microscope image and allows different markers to be laid down over apoptotic, mitotic or normal cells by hand. The system retains a cumulative score of the numbers of each cell type counted, together with the co-ordinates of each individually marked cell irrespective of subsequent movement of the microscope stage or changes in power of the objective lens. Extremely accurate quantification of the various cell types is thus possible for each section studied as areas can be circumscribed at low power (eliminating selection bias), and counted at high power without danger of double scoring. For each animal, three random areas of approximately $47000 \mu \mathrm{m}^{2}$ were scored for the presence of mitotic and apoptotic figures. The sensitivity of detection of trophic events throughout the study was virtually $100 \%$. The error in quantifying the number of normal cells surrounding these events was $\leq 2 \%$. Thus the overall error in estimating the prevalence of trophic events was around 0.001\%. Immunoreactive (rather than trophic) positive and negative cell counts were performed at $400 \times$ magnification in three random areas of approximately $78000 \mu \mathrm{m}^{2}$ per animal. All slides were coded and counted by one observer blind to the experimental protocol.

\section{Statistics}

Results (means \pm S.E.) were expressed as a percentage of the total cell numbers counted for each animal. GraphPad Instat and GraphPad Prism (GraphPad Software, San Diego, CA, USA) were used to perform statistical calculations. Differences between groups were evaluated using one-way ANOVA followed by Tukey-Kramer multiple comparison post tests. $P<0 \cdot 05$ was considered statistically significant.

\section{Results}

Dexamethasone treatment beginning 2 weeks after adrenalectomy induced a highly significant, discrete burst of apoptotic activity within 48 h (Fig. 1b). After two weeks

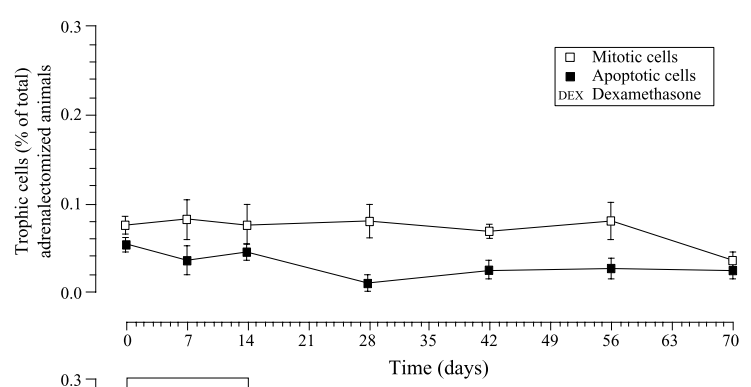

b.
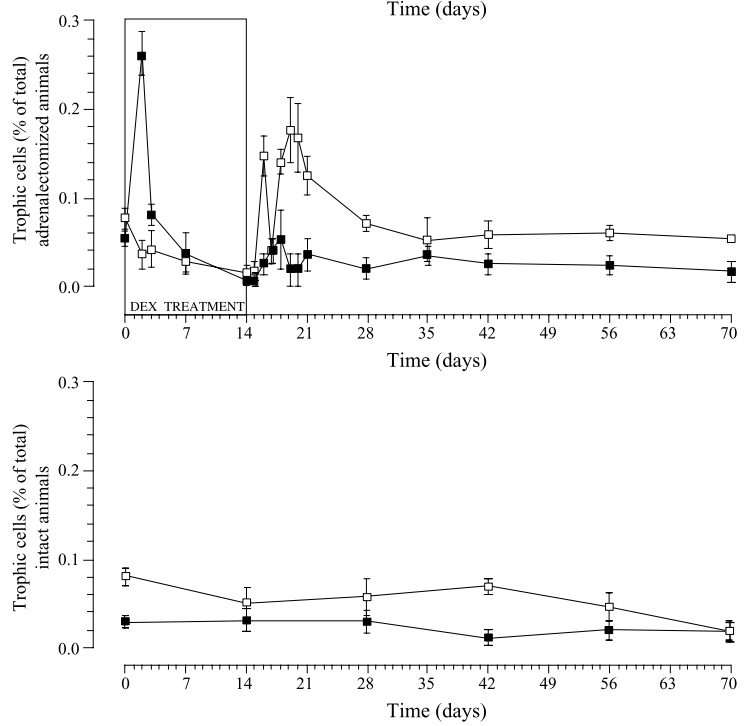

d.

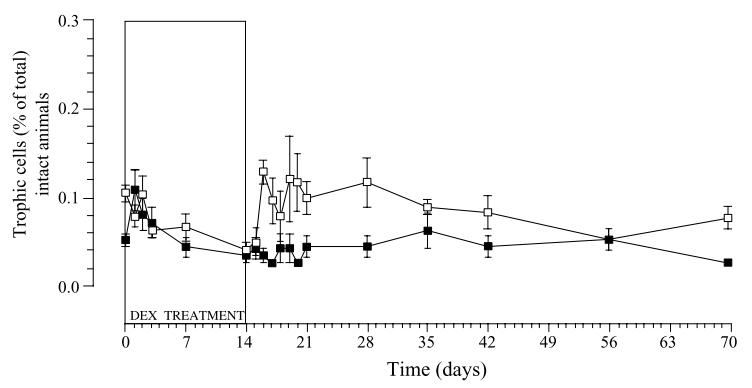

Figure 1 Changes in the prevalence of trophic cells in ( $a$ and b) adrenalectomised and (c and d) intact animals as a percentage of total parenchymal cells. The effects of dexamethasone treatment for 2 weeks in adrenalectomised and intact animals are shown in (b) and (d) respectively. Means \pm S.E. are shown.

of dexamethasone treatment, however, all trophic activity had been reduced to virtually undetectable levels (Fig. 1b: day 14). In comparison, the prevalence of apoptotic and mitotic activity in adrenalectomised age-matched control animals remained unchanged (Fig. 1a: day 14).

\section{The effects of dexamethasone withdrawal on anterior pituitary} trophic activity

A significant and consistent biphasic increase in mitosis began 2 days after withdrawal of dexamethasone and 


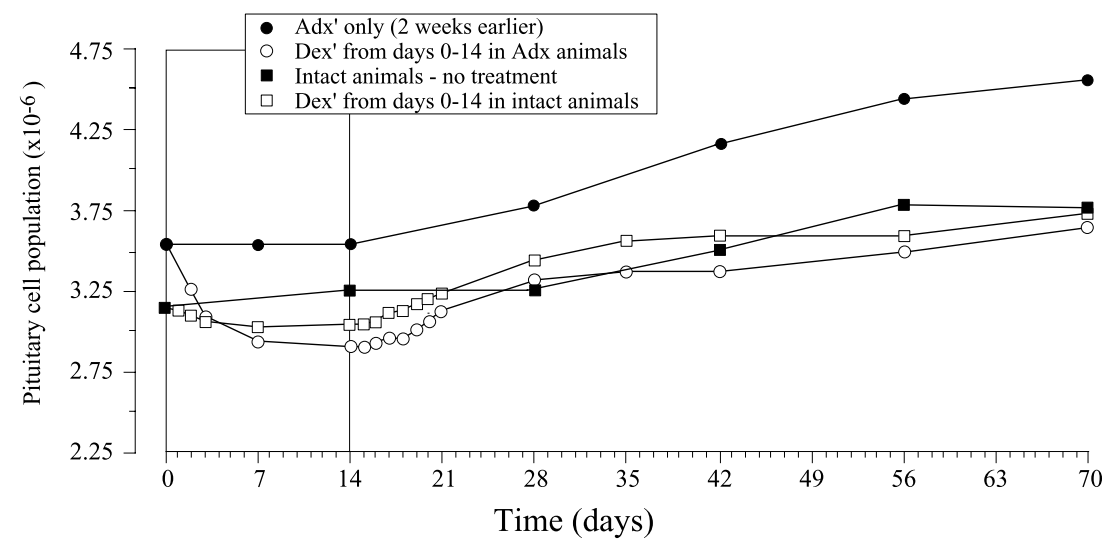

Figure 2 Calculated changes in total pituitary cell populations with time in intact animals and animals adrenalectomised ( $\left(\mathrm{ddx}^{\prime}\right) 2$ weeks previously. The open symbols represent dexamethasone $\left(\right.$ Dex $\left.^{\prime}\right)$-treated groups and the solid symbols represent those treated with vehicle only.

peaked at a prevalence of $0 \cdot 177 \pm 0 \cdot 038 \%$ after 5 days before falling to the level seen in adrenalectomised-only animals by the following week (Fig. 1a and 1b: day 28). Within 3 days of dexamethasone withdrawal (after treatment for 2 weeks) the prevalence of apoptotic figures returned to levels seen in adrenalectomised-only animals and remained constant throughout the remainder of the 8 -week study period (Fig. 1a and 1b).

Trophic changes in intact animals treated with dexamethasone were qualitatively similar to those observed in adrenalectomised animals but much reduced in amplitude (Fig. 1c and 1d).

The effects of dexamethasone withdrawal on anterior pituitary population dynamics

Accurate mitosis and apoptosis prevalence data with precise measurements of the histologically overt duration of each phenomena were used to estimate the composite effects of trophic activity on the pituitary over time. Total pituitary cell numbers in both intact and adrenalectomised animals increase almost in parallel throughout the 10-week study period (solid symbols, Fig. 2). The apparent early absence of population increase in the adrenalectomised group (solid circles) follows two weeks of rapid increase immediately after adrenalectomy (hence the different population of the adrenalectomised groups at 'time zero').

Treatment of adrenal-intact rats with dexamethasone (open boxes) resulted in a small fall in the total pituitary cell population followed by complete recovery within one week of dexamethasone withdrawal (open and solid box symbols). The recovery after dexamethasone withdrawal in adrenalectomised rats (open circles), though rapid, had not offset the dramatic dexamethasone-induced decline in cell numbers by the end of the period of data collection.

Recovery of corticotroph cell numbers following dexamethasone withdrawal

The number of immunocytochemically identifiable corticotrophs in intact animals treated with dexamethasone returned to control levels within one week of withdrawal of dexamethasone (Fig. 3a). Dexamethasone treatment for two weeks significantly reduced the number of ACTHpositive cells in adrenalectomised rats $(5 \cdot 32 \pm 0 \cdot 22 \%$ vs $8.89 \pm 0 \cdot 16 \% ; P<0 \cdot 001 ; n=6$; Fig. 3b). During the subsequent recovery period, the number of identifiable corticotrophs increased steadily to reach a plateau between 6 and 8 weeks later. Although no allowance was made for possible cell size differences between the two treatment groups (which would tend to exaggerate the early differences in cell numbers (Floderus 1944)), corticotroph numbers remained significantly lower in those animals that had received dexamethasone compared with those subjected to adrenalectomy alone after 8 weeks of steroid withdrawal $(7 \cdot 92 \pm 0 \cdot 2 \%$ vs $8 \cdot 66 \pm 0 \cdot 16 \% ; P<0 \cdot 05 ; n=4$; Fig. 3b).

The effect of repeated dexamethasone exposure on trophic activity in the anterior pituitary gland

The ability of the pituitary gland to regenerate a trophically sensitive and dexamethasone-responsive population of cells was investigated by giving sequential courses of dexamethasone. In each case, trophic activity immediately prior to second and third doses of dexamethasone had returned to levels seen in adrenalectomised controls. 
a.
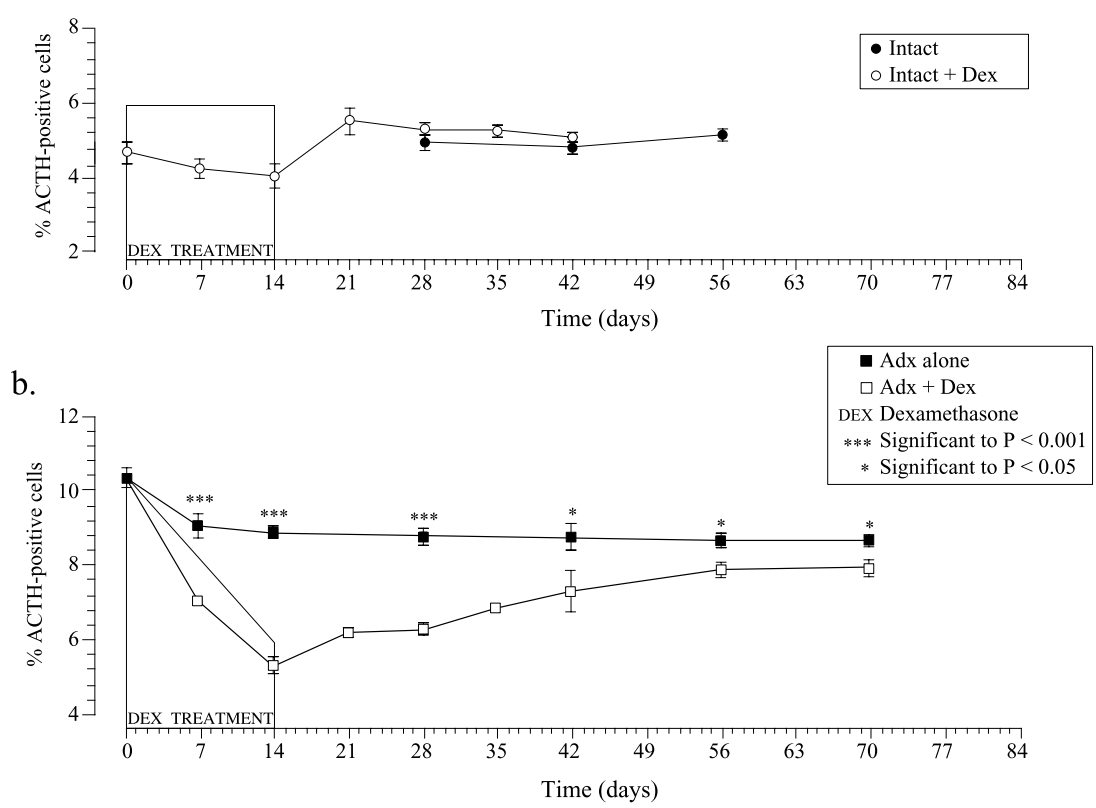

Figure 3 The effects of adrenalectomy $(\mathrm{Adx})$ and dexamethasone treatment on the proportion of immunocytochemically identified corticotroph cells in the pituitary. Means \pm S.E. are shown, $n \geq 4 .{ }^{*} P \leq 0 \cdot 05,{ }^{* * *} P \leq 0 \cdot 001$.

Irrespective of whether the first dose of dexamethasone was given to adrenalectomised (Fig. 4a) or intact rats (before subsequent adrenalectomy: Fig. 4b), second exposure to dexamethasone (6 and 2 weeks after adrenalectomy respectively) once again suppressed mitotic activity and induced a burst of apoptotic activity that again peaked $48 \mathrm{~h}$ after the onset of treatment. However, in each case the amplitude of the apoptotic peak was less than that induced by the first dexamethasone treatment in agematched adrenalectomised controls despite the two- to four-week washout period. When both dexamethasone treatments were given post-adrenalectomy, the peak apoptotic response to the second exposure was significantly reduced $(0 \cdot 146 \pm 0.025 \%$ vs $0.252 \pm 0.028 \%: n=6$ : $P<0 \cdot 01$; Fig. 4a). When the first dexamethasone treatment was given before adrenalectomy, the reduction in peak apoptotic response to second dexamethasone exposure, although reduced, did not quite reach statistical significance $(0 \cdot 18 \pm 0 \cdot 038 \%$ vs $0 \cdot 26 \pm 0 \cdot 017 \% ; n=6$; not significant; Fig. 4b).

When dexamethasone treatment was limited to 3 days (rather than 14 days) and first exposure given 7 days rather than 14 days post-adrenalectomy, the maximum amplitude of apoptotic burst was similar $(0 \cdot 223 \pm 0 \cdot 037 \%$ and $0 \cdot 264 \pm 0.025 \%$ respectively; Figs $4 \mathrm{c}$ and $1 \mathrm{~b}$ ) as was recovery of the anterior pituitary in terms of the prevalence of mitotic figures 7 days later $(0 \cdot 141 \pm 0.023 \%$ vs $0 \cdot 126 \pm 0 \cdot 022 \%$ respectively; $n=6$; Figs $4 \mathrm{c}$ panels II and III, and $1 \mathrm{~b}$ respectively).
The peak of apoptotic activity seen during the second and third exposures to dexamethasone in adrenalectomised rats was again less than that observed during the first exposure to dexamethasone and significantly less than that seen in adrenalectomised age-matched controls exposed to dexamethasone for the first time (Fig. 4c panel III). There was no significant difference in the apoptotic peaks induced by second and third exposures to dexamethasone.

\section{Discussion}

The proliferative response of rat corticotrophs to bilateral adrenalectomy is well recognised (Miskowiak et al. 1987, Gulyas et al. 1991, Taniguchi et al. 1995) and several studies suggest that the concomitant increase in proopiomelanocortin gene transcription is partly due to an absolute increase in the number of cells actively transcribing the gene (Fremeau et al. 1986, Roberts et al. 1987, Autelitano et al. 1989). Our own data and the work of others (Gulyas et al. 1991, Taniguchi et al. 1995) indicate that the post-adrenalectomy surge in pituitary mitotic activity is likely to be derived from both functional corticotrophs and other hormone-secreting cells as well as from more immature or pluripotent stem cells. Indeed, as the rate of increase in the number of corticotrophs following dexamethasone withdrawal is lower than that measured after adrenalectomy despite a similar increase in mitosis (Nolan et al. 1998), populations of pituitary cells 
a.

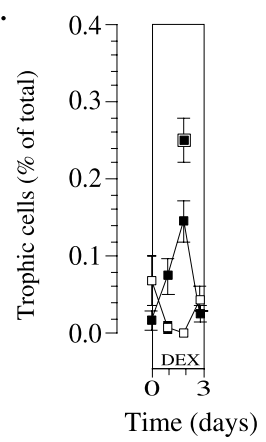

b.

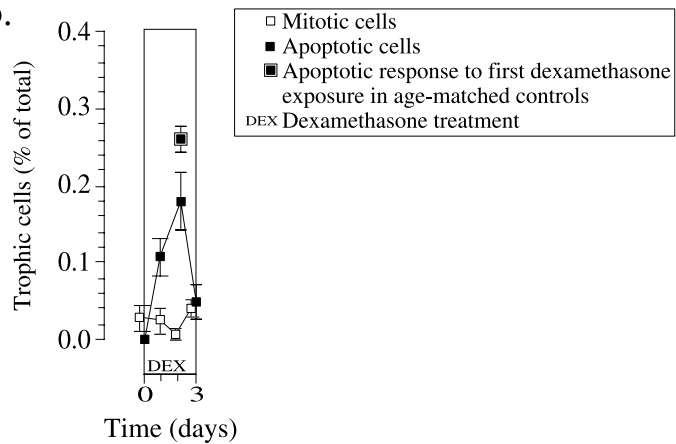

c.

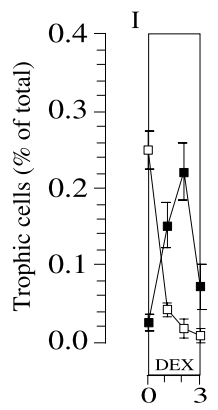

II
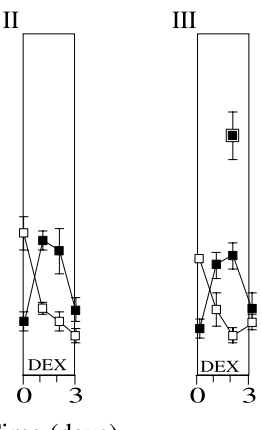

Figure 4 The trophic response to repeated exposures to dexamethasone. (a) Animals were adrenalectomised 6 weeks previously and had had a 2-week course of dexamethasone starting 2 weeks after adrenalectomy. The effects of a second 3-day exposure to dexamethasone, 14 days after the end of the first course are shown. (b) Intact animals received a 2-week course of dexamethasone and were adrenalectomised 2 weeks later. The effects of a second 3-day exposure to dexamethasone 2 weeks after adrenalectomy are shown. (c) The responses to the first, second and third 3-day exposures to dexamethasone (I, II and III respectively) starting 1 week after adrenalectomy with 1 -week recovery periods between each treatment are shown.

other than immunocytochemically identifiable corticotrophs must contribute to the mitotic index at this time.

The increased prevalence of mitotic figure speaks one week after adrenalectomy and returns to preoperative levels after two weeks. By three weeks after adrenalectomy the mean mitotic to apoptotic ratio is virtually identical to that seen in age-matched controls. The proportion of immunologically identifiable corticotrophs also remains constant from three weeks after adrenalectomy at approximately double that seen in controls. The progressive increase in calculated total population of anterior pituitary cells over 12 weeks in both adrenalectomised and control rats suggests that continued absence of glucocorticoid feedback superimposes a new equilibrium on essentially normal pituitary growth.

In both adrenalectomised and intact rats, the prevalence of trophic events and thus cell turnover in the anterior pituitary gland returns to control levels within the first week of withdrawal of long-term, supraphysiological dexamethasone treatment. The prevalence of mitotic events increases in a highly reproducible but inexplicable biphasic pattern immediately after withdrawal of dexamethasone in adrenalectomised animals. Nevertheless, 8 weeks later, the number of immunocytochemically identifiable corticotrophs and the predicted total pituitary cell population remain below baseline. Thus the increased ratio of mitotic to apoptotic activity that occurs immediately after dexamethasone withdrawal in this group of animals is not sustained for sufficient time to allow full recovery of pituitary cell populations within the time period studied.

The apoptotic response to a second dose of dexamethasone after one, two or four weeks recovery was reduced, indicating that complete restoration of a dexamethasoneresponsive cell population does not appear to occur. Importantly, as the reduction in amplitude of the apoptotic burst induced by the second exposure to dexamethasone is the same irrespective of whether the first dose of dexamethasone was given to intact or adrenalectomised animals, the effects on the mitotic population feeding the 
trophically sensitive population are similar. Incidentally, these findings also suggest that the effects of glucocorticoids in adrenalectomised rats is a physiologically relevant model of trophic responsiveness in the pituitary of intact animals, despite being an extreme stimulus. The apoptotic burst seen in the pituitary glands of adrenalectomised rats given three short courses of dexamethasone at 7-day intervals was reduced to the same extent as that seen after two 14-day treatments separated by a 14-day recovery period. There was no additional reduction in magnitude of the apoptotic response between the second and the third dose of dexamethasone, again emphasising that a small subset of anterior pituitary cells deleted by first exposure to dexamethasone appears not to be replaced (at least within the 8 week time frame of the present study).

Persistent traces of dexamethasone are unlikely to account for the reduction in amplitude of apoptotic bursts after first exposure as the effect is independent of the timing of the second dose (7, 14 or 28 days) and our own further studies have shown that under some circumstances, pre-treatment with dexamethasone can actually increase the apoptotic response to second exposure within the same time frame. Furthermore, a significant increase in pituitary mitotic activity occurs within $48 \mathrm{~h}$ of dexamethasone withdrawal and others have shown that secretory parameters are mostly normalised within 3-7 days (Nicholson et al. 1984, Calogero et al. 1990, Huang 1994).

The stimulus of adrenalectomy followed by supraphysiological dexamethasone treatment, withdrawal and re-treatment causes a series of trophic responses within the anterior pituitary gland that result in a prolonged recovery phase with respect to corticotroph cell number, total anterior pituitary cell population and the total proliferative potential of a subpopulation of dexamethasone-sensitive cells. In intact animals, the trophic responses to dexamethasone treatments and withdrawal may be less obvious numerically, but result in deletion of the same subset of cells, thus predisposing the pituitary gland to a sub-optimal proliferative response to subsequent stimuli and perhaps contributing to the delayed functional recovery of the HPA axis following glucocorticoid withdrawal.

\section{Acknowledgement}

We are grateful to the Wellcome Trust for providing financial support for this project.

\section{References}

Autelitano DJ, Blum M \& Roberts JL 1989 Changes in rat pituitary nuclear and cytoplasmic pro-opiomelanocortin RNAs associated with adrenalectomy and glucocorticoid replacement. Molecular and Cellular Endocrinology 66 171-180.

Avgerinos PC, Chrousos GP, Nieman LK, Oldfield EH, Loriaux DL \& Cutler GBJ 1987 The corticotropin-releasing hormone test in the postoperative evaluation of patients with Cushing's syndrome. Journal of Clinical Endocrinology and Metabolism 65 906-913.

Brugal G, Cye R, Krief B, Chassery J-M, Tanke H \& Tucker JH 1992 HOME: highly optimized microscope environment. Cytometry 13 109-116.

Calogero AE, Kamilaris TC, Johnson EO, Tartaglia ME \& Chrousos G 1990 Recovery of the rat hypothalamic-pituitary-adrenal axis after discontinuation of prolonged treatment with the synthetic glucocorticoid agonist dexamethasone. Endocrinology 127 1574-1579.

Carella MJ, Srivastava LS, Gossain VV \& Rovner DR 1993 Hypothalamic-pituitary-adrenal function one week after a short burst of steroid therapy. Journal of Clinical Endocrinology and Metabolism 76 1188-1191.

Floderus S 1944 Untersuchungen über den Bau der menschlichen Hypophyse mit besonderer Berucksichtigung der quantitativen mikromorphologischen Verhä ltnisser. Acta Pathologica Microbiologica Scandinavica Supplement $\mathbf{5 3} 276$.

Ford LR, Willi SM, Hollis BW \& Wright NM 1997 Suppression and recovery of the neonatal hypothalamic-pituitary-adrenal axis after prolonged dexamethasone therapy. Journal of Pediatrics 131 722-726.

Fremeau RT, Lundblad JR, Pritchett DB, Wilcox JN \& Roberts JL 1986 Regulation of pro-opiomelanocortin gene transcription in individual cell nuclei. Science 234 1265-1269.

Graber AL, Ney RL, Nicholson WE, Island DP \& Liddle GW 1965 Natural history of pituitary-adrenal recovery following long-term suppression with corticosteroids. Journal of Clinical Endocrinology and Metabolism 25 11-16.

Gulyas M, Pusztai L, Rappay G \& Makura GB 1991 Pituitary corticotrophs proliferate temporarily after adrenalectomy. Histochemistry 96 185-189.

Harrison BD, Rees LH, Cayton RM \& Nabarro JD 1982 Recovery of hypothalamo-pituitary-adrenal function in asthmatics whose oral steroids have been stopped or reduced. Clinical Endocrinology 17 109-118.

Huang T-S 1994 Corticotropin secretagogues facilitate recovery of the hypothalamus-pituitary-adrenal axis suppressed by prolonged treatment with dexamethasone. Metabolism 43 544-548.

Livanou T, Ferriman D \& James VHT 1967 Recovery of hypothalamo-pituitary-adrenal function after corticosteroid therapy. Lancet 856-860.

Miskowiak B, Stachosiak A, Zabel M \& Malendowicz LK 1987 Studies on hypothalamo-pituitary corticoliberin system. IV. Quantitative changes in ACTH-immunoreactive anterior pituitary cells evoked by long-term intraventricular CRF administration and adrenalectomy. Experimental and Clinical Endocrinology $\mathbf{9 0}$ 178-184.

Nicholson S, Campbell E, Torrellas A, Beckford U, Altaher R, Sandford R, Scraggs R, Gillham B \& Jones M 1984 Recovery of the hypothalamo-pituitary-adrenocortical axis in the rat after long-term dexamethasone treatment. Neuroendocrinology 39 343-349.

Nolan LA, Kavanagh E, Lightman SL \& Levy A 1998 Anterior pituitary cell population control: basal cell turnover and the effects of adrenalectomy and dexamethasone treatment. Journal of Neuroendocrinology 10 207-215.

Nolan LA, Lunness HR, Lightman SL \& Levy A 1999 The effects of age and spontaneous adenoma formation on trophic activity in the rat pituitary gland: a comparison with trophic activity in the human pituitary and in human pituitary adenomas. Journal of Neuroendocrinology 11 393-401.

Roberts JL, Lundblad JR, Eberwine JH, Fremeau RT, Salton SRJ \& Blum M 1987 Hormonal regulation of POMC gene expression in pituitary. Annals of the New York Academy of Sciences 512 275-285.

Schlaghecke R, Kornely E, Santen RT \& Ridderskamp P 1992 The effect of long-term glucocorticoid therapy on pituitary-adrenal responses to exogenous corticotropin-releasing hormone. New England Journal of Medicine 326 226-230. 
Schurmeyer TH, Tsokos GC, Avgerinos PC, Balow JE, D’Agata R, Loriaux DL \& Chrousos GP 1985 Pituitary-adrenal responsiveness to corticotropin-releasing hormone in patients receiving chronic, alternate-day glucocorticoid therapy. Journal of Clinical Endocrinology and Metabolism $6122-27$.

Shu S, Ju G \& Fan L 1988 The glucose oxidase-DAB-nickel method in peroxidase histochemistry of the nervous system. Neuroscience Letters 85 169-171.
Taniguchi Y, Tamatani R, Yasutaka S \& Kawarai Y 1995

Proliferation of pituitary corticotrophs following adrenalectomy as revealed by immunocytochemistry combined with

bromodeoxyuridine-labeling. Histochemistry 103 127-130.

Received 9 October 2000

Accepted 5 January 2001 